Published in final edited form as:

Am Heart J. 2019 August ; 214: 1-8. doi:10.1016/j.ahj.2019.04.013.

\title{
De-escalation of Antianginal Medications after Successful Chronic Total Occlusion Percutaneous Coronary Intervention: Frequency and Relationship with Health Status
}

\author{
Mohammed Qintar, MD ${ }^{1,2}$, Taishi Hirai, MD ${ }^{3}$, Suzanne V. Arnold, MD MHA ${ }^{1,2}$, Justin Sheehy, \\ MD $^{1,2}$, James Sapontis, MBBCh, FRACP ${ }^{4}$, Phil Jones, MS $^{1,2}$, Yuanyuan Tang, PhD ${ }^{1}$, William \\ Lombardi, MD $^{5}$, Dimitri Karmpaliotis, MD $^{6}$, Jeffery Moses, $\mathbf{M D}^{6}$, Christian Patterson, BS ${ }^{1}$, \\ William J. Nicholson, MD ${ }^{7}$, David J. Cohen, MD MSc ${ }^{1,2}$, John A. Spertus, MD MPH ${ }^{1,2}$, J. \\ Aaron Grantham, MD ${ }^{1,2}$, Adam C. Salisbury, MD MSc ${ }^{1,2}$ \\ ${ }^{1}$ Saint Luke's Mid America Heart Institute; \\ ${ }^{2}$ University of Missouri-Kansas City, Kansas City, MO; \\ ${ }^{3}$ Loyola University, Chicago, IL; \\ ${ }^{4}$ Monash Cardiovascular Research Centre, MonashHeart, Monash Health \& Department of \\ Medicine (SCS at Monash), Monash University, Melbourne, Australia; \\ ${ }^{5}$ University of Washington, Seattle, WA; \\ ${ }^{6}$ Columbia University, New York City, NY; \\ ${ }^{7}$ York Hospital, York, PA
}

\section{Abstract}

Background: Successful CTO PCI can markedly reduce angina symptom burden, but many patients often remain on multiple AAMs after the procedure. It is unclear when, or if, AAMs can be de-escalated to prevent side effects or limit polypharmacy. We examined the association of deescalation of antianginal medications (AAMs) after CTO PCI with long-term health status.

Methods: In a 12-center registry of consecutive CTO PCI patients, health status was assessed at 6 months after successful CTO PCI with the Seattle Angina Questionnaire (SAQ) and the Rose Dyspnea Scale (RDS). Among patients with technical CTO PCI success, we examined the

Corresponding Author: Adam C. Salisbury MD MSc, Saint Luke's Mid America Heart Institute, 4401 Wornall Road, Kansas City, MO 64111, Phone: 816-751-8943, Fax: 816-932-5613, asalisbury@ saint-lukes.org.

Disclosure of potential conflicts of interest. ACS: institutional research grant support from Boston Scientific and Gilead, honoraria or speaking fees from Abiomed and Boston Scientific, and consulting fees from Medtronic. JAG: speaking fees and honoraria from Boston Scientific, Abbott Vascular, and Asahi Intecc, institutional research grant support from Boston Scientific, institutional educational grant support from Abbott Vascular, Vascular Solutions, Boston Scientific, and Asahi Intecc, a part time employee of Corindus Vascular Robotics. JS: speaking fees and honoraria from Boston Scientific and Abbott Vascular. WL: speaking fees and honoraria from Boston Scientific, Abbott Vascular, and Abiomed, consultancy for Vascular Solutions, Abbott Vascular, Boston Scientific, Abiomed, and Roxwood Medical. He has Equity in Roxwood Medical and Bridgepoint Medical. His wife is an employee of Spectranetics. DK: reports speaking fees, honoraria, and consulting fees from Abbott Vascular, Boston Scientific, and Medtronic. DJC: institutional research grant support from Boston Scientific, Abbott Vascular, and Medtronic and consulting fees from Medtronic and Abbott Vascular. JAS: research grants from Lilly and Abbott Vascular. He has served as a consultant for Novartis, Amgen, Bayer and United Healthcare. He owns the copyright to the SAQ, and has an equity interest in Health Outcomes Sciences. The remaining authors have no relevant relationships to disclose. 
association of AAM de-escalation with 6-month health status using multivariable models adjusting for $\mathrm{c}$ revascularization completeness and predicted risk of post-PCI angina (using a validated risk model). We also examined predictors and variability of AAMs de-escalation.

Results: Of 669 patients with technical success of CTO PCI, AAM were de-escalated in 276 (35.9\%) patients at 1 month. Patients with AAM de-escalation reported similar angina and dyspnea rates at 6 months compared with those whose AAM were reduced (any angina: 22.5\% vs. $20 \%, \mathrm{p}=0.43$; any dyspnea: $51.8 \%$ vs. $50.1 \%, \mathrm{p}=0.40$ ). In a multivariable model adjusting for complete revascularization and predicted risk of post-PCI angina, de-escalation of AAMs at 1 month was not associated with an increased risk of angina, dyspnea or worse health status at 6 months.

Conclusions: Among patients with successful CTO PCI, de-escalation of AAMs occurred in $\sim 1 / 3$ of patients at 1 month and was not associated with worse long-term health status.

\section{Keywords}

chronic total occlusion; antianginal medications; percutaneous coronary intervention; angina; dyspnea; quality of life

Patients with coronary artery chronic total occlusions (CTOs) often present a significant challenge to cardiologists managing their angina symptoms. These patients frequently suffer from longstanding angina and rely on multiple antianginal medications (AAM). ${ }^{1}$ Current guidelines strongly endorse medical therapy, ${ }^{2,3}$ including maximally tolerated AAMs, as first-line therapy for management of chronic stable angina in patients with obstructive coronary artery disease (CAD). However, many patients continue to experience lifestylelimiting angina despite up-titration of these medications and are ultimately referred for coronary revascularization. ${ }^{4}$ Despite this clear guidance on maximizing medications prior to revascularization, there is no clear guidelines on best practices regarding continuation or deescalation of AAM therapy after successful revascularization. Since patients with complex coronary disease, such as CTOs, may have residual lesions or small vessel disease, it is possible that de-escalation of AAMs could lead to rebound angina. Moreover, given that many AAMs (e.g. nitrates, ranolazine, calcium channel blockers) are generally used for symptom relief and not mortality reduction, and given that these medications can be associated with adverse effects ${ }^{5-8}$ and that patients often become frustrated with complex medication regimens, a thoughtful approach to de-escalation of medications that are no longer needed for symptom control should be an important component of the post-PCI treatment plan.

Given the additional technical challenges of CTO percutaneous coronary intervention (PCI) and the limited number of sites that offer this as an option for revascularization, attempts at medical management of angina are often even more intense in patients with CTOs prior to revascularization. ${ }^{1,9-11}$ Furthermore, there are some data that empiric de-escalation of AAMs at the time of PCI may be associated with greater symptom burden, especially in patients with incomplete revascularization. ${ }^{12}$ To help support providers in their understanding of when it is safe to attempt AAM de-escalation after CTO PCI, we used the Outcomes, Patient Health Status, and Efficiency IN Chronic Total Occlusion Hybrid 
Procedures (OPEN-CTO) Registry, a contemporary 12-center registry of CTO PCI, to describe the frequency of AAM de-escalation after CTO PCI and its association with patients' symptoms.

\section{METHODS}

\section{Study design and population.}

The OPEN CTO Registry is a prospective, single-arm registry that enrolled patients with CTOs who underwent attempted CTO PCI at 12 US sites. ${ }^{13}$ Eligible patients were $\geq 18$ years of age, had symptoms suggestive of ischemic heart disease, and had CTOs of at least 1 epicardial coronary artery (defined as TIMI grade 0 flow distal to the occlusion with a presumed duration of total occlusion of at least 3 months). CTO PCI operators were required to have performed at least 100 CTO PCI procedures over a minimum of 2 years before participating in OPEN CTO. Technical success of the procedure was defined as $<50 \%$ residual stenosis and TIMI 2 or 3 flow without any side branch occlusion. Physiologically complete revascularization was defined by the operators as successful treatment of all physiologically significant lesions at the time of the index procedure. Because the focus of this study was on the frequency and outcomes of reducing AAM treatment, we restricted the cohort to those whose index procedure was technically successful. Also, patients who were on no anti-anginal medications at baseline were excluded. Each participating site obtained Institutional Research Board approval, and all patients provided informed consent.

\section{Assessment of health status.}

Angina and dyspnea were assessed at baseline and at 6 months after CTO PCI with the Seattle Angina Questionnaire (SAQ) and Rose dyspnea scale (RDS), respectively. The SAQ is a 19-item questionnaire with a 4-week recall period that measures 5 domains of health in patients with CAD: angina frequency (SAQ AF), angina stability, quality of life (QoL), physical limitation (PL), and treatment satisfaction. ${ }^{14,15}$ The SAQ Summary Scale (SAQ SS) integrates the SAQ AF, SAQ QoL, and SAQ PL into a single summary score. ${ }^{16}$ Domain and summary scores range from 0 to 100 , with higher scores indicating fewer symptoms and better quality of life. A mean change of $\sim 5$-points is considered clinically meaningful. ${ }^{14,15}$ The SAQ has undergone extensive reliability and validity testing, ${ }^{15,17}$ and is associated with long-term survival, hospitalization for acute coronary syndromes, and healthcare utilization among patients with chronic CAD. ${ }^{18}$ The SAQ AF domain captures the frequency of angina and sublingual nitroglycerin use and has been shown to correlate closely with daily angina diaries. ${ }^{19}$ The primary outcome for this study was the presence of any angina at 6 months after CTO PCI, which was defined as a SAQ AF score $<100 .{ }^{20}$ We also examined the SAQ AF, SAQ QoL, SAQ PL, and SAQ SS scores as continuous variables.

The RDS is a 4-item questionnaire with a 1-month recall period that assesses patients' level of dyspnea with common activities (Supplemental Table 1). ${ }^{21}$ For each patient, the highest limitation associated with dyspnea was designated as the RDS score, such that RDS scores range from 0 to 4 , with 0 indicating no dyspnea and 4 indicating dyspnea with ordinary activities of washing and dressing. The RDS has been validated in patients with CAD and 
shown to be associated with quality of life, rehospitalization, and long-term survival in patients with $\mathrm{CAD}{ }^{22}$

\section{Definition of de-escalation of AAM.}

AAM de-escalation in early follow-up was defined as: 1) being on fewer AAMs at 1 month compared with admission or 2) a decrease in AAM dose, if there was no change in the number of AAMs taken. To define a change in AAM dose, we determined the maximum recommended dose of each AAM for treating angina and then calculated the patient's $\%$ of maximum dose. The proportion of maximum dose being taken for each agent was compared at admission and at 1 month for each patient, and each AAM class of medication was assumed to have a similar efficacy in treatment of angina, consistent with prior literature. 23,24 This allowed us to compare changes in medications within class (e.g., change from diltiazem to amlodipine) and changes in medication classes (e.g., change from carvedilol to isosorbide). For our primary analysis, any de-escalation of medications was considered significant. Patients who were on no anti-anginal medications at baseline were excluded.

\section{Statistical methods.}

Demographic and clinical characteristics were compared among patients whose AAM were de-escalated compared to those whose AAM were not de-escalated at 1 month using $t$-tests for continuous variables and chi-square tests for categorical variables. The proportion of patients who reported angina, and the unadjusted health status scores at baseline and 6 months after CTO PCI, were compared among groups using chi-square tests and $t$-tests, respectively. To examine the independent association of AAM de-escalation (vs. continuation or escalation of AAM regimens) with angina, health status, and dyspnea at 6 months after successful CTO PCI, we constructed a hierarchical, multivariable logistic regression model to account for clustering by site and potential confounding. These models included the patient's predicted risk of post-CTO PCI angina (based on pre-procedural factors) and completeness of revascularization. To determine the patient's predicted risk of post-CTO PCI angina, we used a previously published model that was developed to predict residual angina after conventional PCI and was subsequently validated in CTO PCI patients. 25 This model included the following pre-procedural factors: age, self-reported avoidance of care due to cost, depression, number of AAM at admission, self-reported pain or discomfort [question from the EuroQol-5D], SAQ AF, and SAQ QoL. ${ }^{725}$ Since beta-blockers are a corner stone treatment for patients with heart failure and acute myocardial infarction and might not be a target for de-escalation despite optimal angina symptom control, sensitivity analyses excluding all users of beta-blockers and patients with history of heart failure using beta-blcokers were performed.

Next, we sought to describe predictors of AAMs de-escalation and variability in rate of AAMs de-escalation across participating sites. To accomplish this goal, we fit a hierarchical multivariable logistic regression model and included participating site as a random effect, to account for the clustering of patients within each participating site. The outcome of this model was any de-escalation of AAMs, and the independent variables were selected a priori based on clinical judgment and literature review while balancing against risk of model overfitting. Variables included the following: age, sex, prior percutaneous coronary intervention, 
prior coronary artery bypass graft surgery, hypertension, diabetes mellitus, estimated glomerular filtration rate, history of heart failure, predicted risk of angina (based on the previously validated model as mentioned above), physiologically complete revascularization, left ventricular ejection fraction, number of antianginal medications at baseline, SAQ AF at baseline, SAQ AF at 1 month follow-up, RDS score at baseline and RDS score at 1 month. Furthermore, variability in AAMs de-escalation rates among participating sites was studied using the median odds ratio, which estimates the average relative difference in two hypothetically identical patients receiving AAMs de-escalation if presenting to two different sites in the OPEN CTO registry. For example, median odds ratio of 1 suggests no site-level variation in AAMs de-escalation, while an MOR of 2 suggests a 2-fold difference in the odds of receiving AAMs de-escalation comparing two random sites providing care for patients with identical characteristics. Multiple imputation for the missing covariate data (all covariates had $<5 \%$ missing information) was performed using IVEWARE software. All statistical analyses were performed with SAS, version 9.4 (SAS Institute, Inc., Cary, NC).

\section{RESULTS}

\section{Patient characteristics.}

Among 1000 patients enrolled in OPEN CTO who underwent CTO PCI, we excluded 137 patients who had technical CTO PCI failure, 83 patients with missing SAQ AF data at either baseline or follow up, 11 patients with missing AAM data and 73 patient who were not on AAM at baseline. Accordingly, our analytic cohort included 696 patients (Figure 1). Participants' mean age was 65.6 years, $80 \%$ were men, and $90.4 \%$ were white (Table 1). Cardiac comorbidities were common, with prior PCI in $64.2 \%$, prior bypass graft surgery in $36.8 \%$, prior myocardial infarction $47.1 \%$, prior PAD in $17.1 \%$, prior heart failure in $21.0 \%$, hypertension in $87.3 \%$, and diabetes in $38.2 \%$. Complete physiologic revascularization was achieved in $85.0 \%$ of patients. Post CTO PCI MI occurred in $2.0 \%$ of patients.

\section{Baseline angina and AAM.}

At admission prior to CTO PCI, the mean SAQ AF score was $69.0 \pm 27.3$ and the mean RDS score was $2.0 \pm 1.4$, and all of patients were on at least 1 AAM with a mean $( \pm \mathrm{SD})$ of $1.8 \pm$ 0.8 AAM per patient. Specific AAMs used were beta-blockers in $92.4 \%$, long-acting nitrates in $44.4 \%$, calcium-channel blockers in $25.9 \%$, and ranolazine in $15.2 \%$ of patients (Table 1 ). At 1 month, $92.4 \%$ of patients were on at least 1 AAM with a mean $( \pm \mathrm{SD})$ of $1.6 \pm 0.7$ AAM per patient. From admission to 1 month, AAMs were de-escalated in 276 patients (39.6\%). The demographic and clinical characteristics of patients whose AAMs were deescalated were generally similar to those whose AAMs were not de-escalated (Table 1). However, patients whose AAMs were de-escalated reported, on average, a greater burden of baseline angina and worse quality of life at baseline and had a higher predicted risk of residual angina after PCI.

\section{6-month angina and health status.}

In unadjusted analysis, the proportion of patients who reported angina and dyspnea at 6 months after CTO PCI was similar to those whose AAM were de-escalated vs. not deescalated (Any angina: $22.5 \%$ vs. $20.0 \%$, $\mathrm{p}=0.434$; any dyspnea: $51.8 \%$ vs. $50.1 \%$, $\mathrm{p}=0.40$; 
Figure 2). The SAQ AF domain scores at 6 months were numerically lower in patients with AAM de-escalation (vs. no descalation), but the difference was not clinically meaningful. The SAQ PL, SAQ QoL, SAQ SS and RDS were all similar between the two groups (Table 1 and 2). In the multivariable model that adjusted for the patient's pre-procedural risk for postPCI angina, de-escalation of AAM at 1 month was not associated with long term angina, angina-related health status or dyspnea (Figure 3). Sensitivity analyses excluding all users of beta-blockers and patients with history of heart failure using beta-blockers showed similar results to the primary analysis and are available upon request.

\section{Variability in AAMs de-escalation among OPEN CTO sites.}

The overall rate of AAMs de-escalation across the 12 OPEN CTO sites ranged from 1 to $15 \%$. After adjusting for clinical characteristics, the median odds ratio for variability across sites was 1.02 , indicating no meaningful site-level variability of AAMs de-escalation.

\section{Predictors of AAMs de-escalation.}

Significant predictors of AAMs de-escalation among patients with technically successful CTO PCI included worse SAQ AF at baseline ( $\mathrm{p}=0.03$ ) and higher number of AAMs at baseline $(\mathrm{p}<0.001)$ (Supplemental Table 2).

\section{Sensitivity analyses.}

A sensitivity analysis was conducted regarding the use of beta-blockers. Results were unchanged and suggest that de-escalation of non-beta-blocker anti-anginals (in case of the presence of an indication for beta-blockers continuation) could be considered.

\section{DISCUSSION}

In a large, multicenter registry of patients undergoing CTO PCI, we found that $39 \%$ of patients had their AAM regimen de-escalated within 1 month after successful CTO PCI. Importantly, AAM de-escalation at 1 month was not associated with worse follow-up angina, dyspnea and health status. No site-level variability in AAMs de-escalation was detected, and only baseline angina frequency and the number of AAMs were significant predictors of AAMs de-escalation. Our findings suggest that AAM de-escalation after successful CTO PCI can be safely considered, and is not associated with increased risk of angina, dyspnea or worse health status. Moreover, the lack of variability in de-escalation practice could suggest clinical inertia may exist to continue these medications after the procedure. Further study is needed to determine the ideal timing, barriers to de-escalation and strategy of AAM de-escalation following successful CTO PCI.

Prior studies have generally focused on exploring the factors associated with angina after PCI. ${ }^{26-29}$ However, scarce data exist on the management of AAMs after PCI, and the impact of changes in AAM regimens on long-term ischemic symptoms. We recently published data on AAM de-escalation after conventional PCI and showed that $11 \%$ of patients had empiric AAM de-escalation at discharge after PCI. ${ }^{12}$ We found that this empiric de-escalation was associated with increased risk of residual angina after PCI and worse long-term health status; however, this was primarily driven by patients with incomplete revascularization. 
AAM de-escalation was not associated with residual symptoms among those who underwent complete revascularization, consistent with the results of the present analysis. Prior literature showed that AAM escalation with ranolazine has not been shown to reduce risk of angina in patients with incomplete revascularization (sometimes due to untreated CTO lesion) ${ }^{30}$. Our data demonstrates that AAM de-escalation can be safely attempted in a population of patients undergoing CTO PCI, particularly among patients with successful PCI who received complete revascularization. These findings are also consistent with the recently published EURO CTO trial, a randomized controlled trial of CTO PCI with optimal medical therapy vs optimal medical therapy alone. The EURO CTO trial showed that in patients with stable angina and CTO, CTO PCI is superior to medical therapy in angina relief and quality life. ${ }^{31}$

There are no published guidelines, algorithms or recommendations to help guide AAM management post PCI, especially after successful CTO PCI. Often, patients are on multiple $\mathrm{AAM}$ at presentation, and it is unclear how to manage them after successful revascularization. It is not uncommon that patients are symptom free after successful PCI but continue to take potentially unnecessary AAM medications that were prescribed prior to the procedure. In our multi-center registry, the overall rate of AAM de-escalation rates was modest and there was no site-level variability. This could represent strong clinical inertia where providers are less likely to de-escalate therapy despite adequate symptom control post PCI. Although clinical inertia is one possible explanation for the lack of de-escalation, other clinical indications for medications continuation exist; such as treatment of heart failure and resistant hypertension. As AAMs generally do not have a survival benefit in patients with stable CAD (in the absence of other comorbidities, such as systolic heart failure or hypertension), a thoughtful de-escalation of these medications after successful revascularization likely makes sense if no other indication is present to justify continuation of these agents.

In light of the data presented in this study, it appears that it is not unreasonable to attempt to de-escalate AAM after successful CTO PCI, which may help avoid polypharmacy and limit un-necessary adverse effects and costs. ${ }^{32}$ One strategy might be to try to de-escalate AAM therapy in follow-up among patients with successful CTO PCI, especially if they undergo physiologically complete revascularization and they have low predicted risk of post PCI angina. We have shown that de-escalation of AAM in this population is not associated with increased residual angina, dyspnea or worse health status. However, it is important to realize that PCI does not eliminate angina in all patients (residual angina was still reported up to $20 \%$ of patients post successful CTO $\mathrm{PCI}^{33}$ ), and symptom guided AAM management with appropriate follow up is necessary, especially in patients with high predicted risk of post PCI angina and incomplete revascularization.

The findings of our study should be interpreted in consideration of the following potential limitations. First, our data is observational, and residual confounding cannot be excluded in interpretation of these results. Randomized trials testing specific strategies of AAM deescalation would be helpful to clarify best practices regarding AAM de-escalation. Second, we defined change in AAM regimens assuming equal antianginal properties for all AAM classes. While this approach was based upon prior studies that have shown no difference in efficacy of angina relief across AAM classes, ${ }^{23,24}$ we cannot exclude differences in 
responses to medications in individual patients. Third, the reasons for AAM de-escalation were not collected in this registry, and these could be physician- or patient-driven. While we do not believe this would necessarily influence our findings, further studies of the reasons behind de-escalation could provide insights to help better understand patterns of AAM deescalation and determine best practices. Likewise, no data was collected on the adverse effects of anti-anginal medications that would provide further insights into the need for AAM de-escalation in select cases. Fourth, complete physiologic revascularization was defined based on operator report, and their assessment and was not adjudicated independently. Finally, beta-blockers are believed to have mortality benefit in patients with depressed left ventricular ejection fraction ${ }^{34}$ and ACS patients ${ }^{35}$, and physicians might be hesitant to de-escalate these medications. However, our sensitivity analysis excluding deescalation of BB showed similar results to the primary analysis, suggesting that deescalating other non-BB medications similarly do not affect health status long term post successful CTO PCI.

In conclusion, we report that over 1 in 3 of patients undergo AAM de-escalation in early follow-up after successful CTO PCI. Importantly, this was not associated with increased risk of residual angina, dyspnea, or worse health status at 6 months after successful CTO PCI. Although current guidelines do not provide guidance on when or if to de-escalate these medications that were started for symptom relief, our findings support considering attempts to de-escalate AAMs after successful CTO PCI. This strategy can simplify patients' medication regimens and avoid side effects from medications that are no longer providing benefit to the patient. Future research is needed to understand the ideal timing and strategy of AAM de-escalation post-PCI and its association with health status.

\section{Supplementary Material}

Refer to Web version on PubMed Central for supplementary material.

\section{ACKNOWLEDGEMENTS}

Funding sources. This project was funded by an unrestricted grant from Gilead Sciences. Dr. Qintar is supported by The National Heart, Lung, and Blood Institute of the National Institutes of Health under Award Number T32HL110837. OPEN CTO registry was funded by unrestricted grant support from Boston Scientific. All data collection, data analyses, the preparation of the manuscript, and the decision to submit the manuscript for publication were conducted independently of the study sponsors.

\section{REFERENCES}

1. Sapontis J, Salisbury AC, Yeh RW, et al. Early Procedural and Health Status Outcomes After Chronic Total Occlusion Angioplasty: A Report From the OPEN-CTO Registry (Outcomes, Patient Health Status, and Efficiency in Chronic Total Occlusion Hybrid Procedures). JACC Cardiovasc Interv. 814 2017;10(15):1523-1534. [PubMed: 28797429]

2. Fihn SD, Blankenship JC, Alexander KP, et al. 2014 ACC/AHA/AATS/PCNA/SCAI/STS focused update of the guideline for the diagnosis and management of patients with stable ischemic heart disease: a report of the American College of Cardiology/American Heart Association Task Force on Practice Guidelines, and the American Association for Thoracic Surgery, Preventive Cardiovascular Nurses Association, Society for Cardiovascular Angiography and Interventions, and Society of Thoracic Surgeons. Journal of the American College of Cardiology. 1104 2014;64(18):1929-1949. [PubMed: 25077860] 
3. Coronary Revascularization Writing G, Patel MR, Dehmer GJ, et al. ACCF/SCAI/STS/AATS/AHA/ ASNC/HFSA/SCCT 2012 appropriate use criteria for coronary revascularization focused update: a report of the American College of Cardiology Foundation Appropriate Use Criteria Task Force, Society for Cardiovascular Angiography and Interventions, Society of Thoracic Surgeons, American Association for Thoracic Surgery, American Heart Association, American Society of Nuclear Cardiology, and the Society of Cardiovascular Computed Tomography. The Journal of thoracic and cardiovascular surgery. 4 2012;143(4):780-803. [PubMed: 22424518]

4. Spertus JA, Maron DJ, Cohen DJ, et al. Frequency, predictors, and consequences of crossing over to revascularization within 12 months of randomization to optimal medical therapy in the Clinical Outcomes Utilizing Revascularization and Aggressive Drug Evaluation (COURAGE) trial. Circ Cardiovasc Qual Outcomes. 7 2013;6(4):409-418. [PubMed: 23838107]

5. Barron AJ, Zaman N, Cole GD, Wensel R, Okonko DO, Francis DP. Systematic review of genuine versus spurious side-effects of beta-blockers in heart failure using placebo control: recommendations for patient information. Int J Cardiol. 109 2013;168(4):3572-3579. [PubMed: 23796325]

6. Rousan TA, Mathew ST, Thadani U. The risk of cardiovascular side effects with anti-anginal drugs. Expert opinion on drug safety. 12 2016;15(12):1609-1623. [PubMed: 27659354]

7. Rayner-Hartley E, Sedlak T. Ranolazine: A Contemporary Review. J Am Heart Assoc. 315 2016;5(3):e003196. [PubMed: 26979079]

8. McDonagh MS, Eden KB, Peterson K. Drug Class Review: Calcium Channel Blockers: Final Report. Portland (OR)2005.

9. Safley DM, Grantham JA, Hatch J, Jones PG, Spertus JA. Quality of life benefits of percutaneous coronary intervention for chronic occlusions. Catheterization and cardiovascular interventions : official journal of the Society for Cardiac Angiography \& Interventions. 1001 2014;84(4):629-634. [PubMed: 24259445]

10. Olivari Z, Rubartelli P, Piscione F, et al. Immediate results and one-year clinical outcome after percutaneous coronary interventions in chronic total occlusions: data from a multicenter, prospective, observational study (TOAST-GISE). Journal of the American College of Cardiology. 521 2003;41(10):1672-1678. [PubMed: 12767645]

11. Cheng AS, Selvanayagam JB, Jerosch-Herold M, et al. Percutaneous treatment of chronic total coronary occlusions improves regional hyperemic myocardial blood flow and contractility: insights from quantitative cardiovascular magnetic resonance imaging. JACC Cardiovasc Interv. 2 2008;1(1):44-53. [PubMed: 19393143]

12. Qintar M, Towheed A, Tang F, et al. The Impact of De-escalation of Antianginal Medications on Health Status After Percutaneous Coronary Intervention. J Am Heart Assoc. 1020 2017;6(10).

13. Sapontis J, Marso SP, Cohen DJ, et al. The Outcomes, Patient Health Status, and Efficiency IN Chronic Total Occlusion Hybrid Procedures registry: rationale and design. Coronary artery disease. 10132016.

14. Spertus JA, Winder JA, Dewhurst TA, Deyo RA, Fihn SD. Monitoring the quality of life in patients with coronary artery disease. The American journal of cardiology. 1215 1994;74(12):1240-1244. [PubMed: 7977097]

15. Spertus JA, Winder JA, Dewhurst TA, et al. Development and evaluation of the Seattle Angina Questionnaire: a new functional status measure for coronary artery disease. Journal of the American College of Cardiology. 2 1995;25(2):333-341. [PubMed: 7829785]

16. Chan PS, Jones PG, Arnold SA, Spertus JA. Development and validation of a short version of the Seattle angina questionnaire. Circulation. Cardiovascular quality and outcomes 9 2014;7(5):640647. [PubMed: 25185249]

17. Babaliaros V, Devireddy C, Lerakis S, et al. Comparison of transfemoral transcatheter aortic valve replacement performed in the catheterization laboratory (minimalist approach) versus hybrid operating room (standard approach): outcomes and cost analysis. JACC Cardiovasc Interv. 8 2014;7(8):898-904. [PubMed: 25086843]

18. Arnold SV, Morrow DA, Lei Y, et al. Economic impact of angina after an acute coronary syndrome: insights from the MERLIN-TIMI 36 trial. Circulation. Cardiovascular quality and outcomes 7 2009;2(4):344-353. [PubMed: 20031860] 
19. Arnold SV, Kosiborod M, Li Y, et al. Comparison of the Seattle Angina Questionnaire With Daily Angina Diary in the TERISA Clinical Trial. Circ Cardiovasc Qual Outcomes. 11 2014;7(6):844850. [PubMed: 25249560]

20. Spertus JA, Salisbury AC, Jones PG, Conaway DG, Thompson RC. Predictors of quality-of-life benefit after percutaneous coronary intervention. Circulation. 1221 2004;110(25):3789-3794. [PubMed: 15596563]

21. Rose GA, Blackburn H. Cardiovascular survey methods. Monogr Ser World Health Organ. 1968;56:1-188.

22. Arnold SV, Spertus JA, Jones PG, Xiao L, Cohen DJ. The impact of dyspnea on health-related quality of life in patients with coronary artery disease: results from the PREMIER registry. Am Heart J. 6 2009;157(6):1042-1049 e1041. [PubMed: 19464415]

23. Heidenreich PA, McDonald KM, Hastie T, et al. Meta-analysis of trials comparing beta-blockers, calcium antagonists, and nitrates for stable angina. Jama. 526 1999;281(20):1927-1936.

[PubMed: 10349897]

24. (UK) RCoP. Stable Angina: Methods, Evidence \& Guidance [Internet]. National Clinical Guidelines Centre (UK). 2011;NICE Clinical Guidelines, No. 126.

25. Qintar M, Hirai T, Patterson C, et al. TCT-496 Predictors of Residual Angina after Chronic Total Occlusion Percutaneous Coronary Intervention: External Validation of an Existing Model. Journal of the American College of Cardiology. 2017;70(18):B205-B206.

26. Weintraub WS, Spertus JA, Kolm P, et al. Effect of PCI on quality of life in patients with stable coronary disease. The New England journal of medicine. 814 2008;359(7):677-687. [PubMed: 18703470]

27. Arnold SV, Jang J-S, Tang F, Graham G, Cohen DJ, Spertus JA. Prediction of Residual Angina After Percutaneous Coronary Intervention. European Heart Journal - Quality of Care and Clinical Outcomes. 2015.

28. Maddox TM, Reid KJ, Spertus JA, et al. Angina at 1 year after myocardial infarction: prevalence and associated findings. Archives of internal medicine. 623 2008;168(12):1310-1316. [PubMed: 18574088]

29. Arnold SV, Masoudi FA, Rumsfeld JS, Li Y, Jones PG, Spertus JA. Derivation and validation of a risk standardization model for benchmarking hospital performance for health-related quality of life outcomes after acute myocardial infarction. Circulation. 121 2014;129(3):313-320. [PubMed: 24163068]

30. Alexander KP, Weisz G, Prather K, et al. Effects of Ranolazine on Angina and Quality of Life After Percutaneous Coronary Intervention With Incomplete Revascularization: Results From the Ranolazine for Incomplete Vessel Revascularization (RIVER-PCI) Trial. Circulation. 105 2016;133(1):39-47. [PubMed: 26555329]

31. Werner GS, Martin-Yuste V, Hildick-Smith D, et al. A randomized multicentre trial to compare revascularization with optimal medical therapy for the treatment of chronic total coronary occlusions. Eur Heart J. 77 2018;39(26):2484-2493. [PubMed: 29722796]

32. Pound P, Britten N, Morgan M, et al. Resisting medicines: a synthesis of qualitative studies of medicine taking. Social science \& medicine. 7 2005;61(1):133-155. [PubMed: 15847968]

33. Longmore RB, Spertus JA, Alexander KP, et al. Angina frequency after myocardial infarction and quality of life in older versus younger adults: the Prospective Registry Evaluating Myocardial Infarction: Event and Recovery study. American heart journal. 3 2011;161(3):631-638. [PubMed: 21392621]

34. Chatterjee S, Biondi-Zoccai G, Abbate A, et al. Benefits of beta blockers in patients with heart failure and reduced ejection fraction: network meta-analysis. Bmj. 1 16 2013;346:f55. [PubMed: 23325883]

35. Bangalore S, Makani H, Radford M, et al. Clinical outcomes with beta-blockers for myocardial infarction: a meta-analysis of randomized trials. The American journal of medicine. 10 2014;127(10):939-953. [PubMed: 24927909] 


\section{KEY POINTS}

\section{Question:}

Can anti-anginal medications (AAM) be de-escalated early after successful chronic total occlusion (CTO) percutaneous coronary intervention (PCI) without worsening health status outcomes?

\section{Findings:}

In a large US CTO PCI registry, patients whose AAM were de-escalated reported similar angina and dyspnea rates at 6 months compared with those whose AAM were not reduced. In a multivariable model, early de-escalation of AAMs was not associated with an increased risk of angina, dyspnea or worse health status at 6 months.

\section{Meaning:}

Among patients with successful CTO PCI, early de-escalation of AAMs can be considered and appears to be safe. 


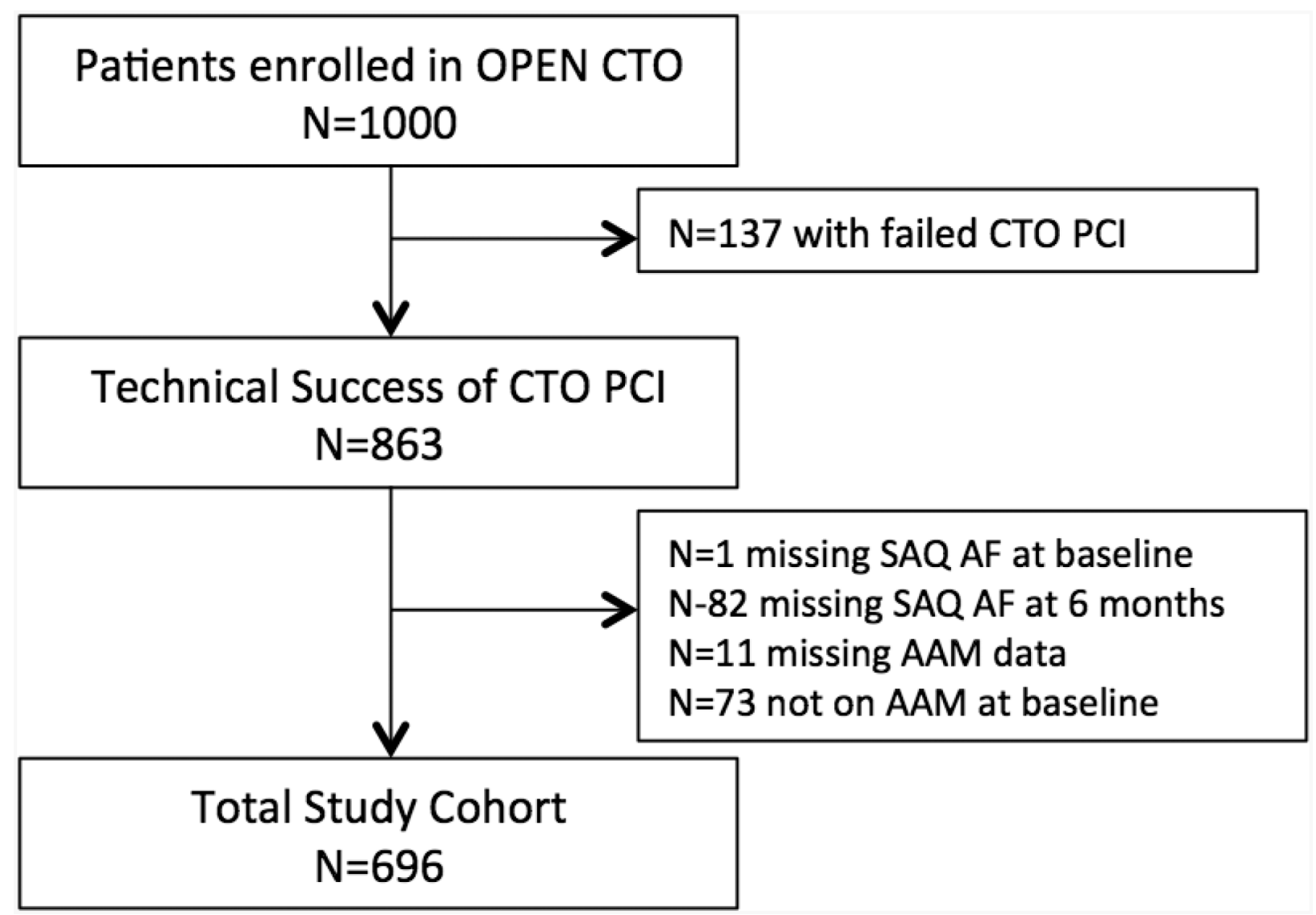

Figure 1. Study patient population.

Flow chart of patients in this study. 


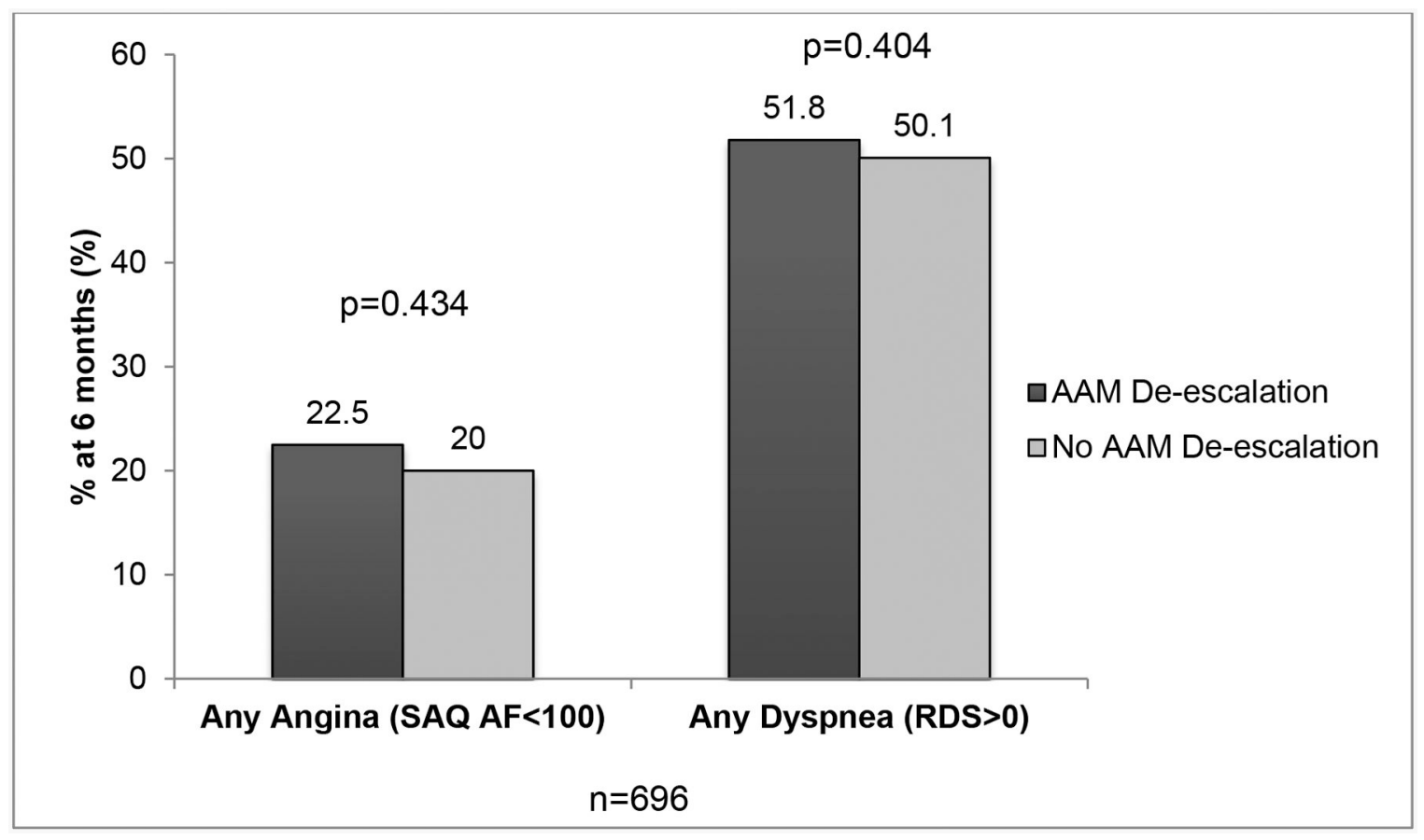

Figure 2. Angina and dyspnea rates at 6 months post PCI.

Unadjusted rates of observed angina and dyspnea according whether AAMs were deescalated at 1 month after successful CTO PCI. 


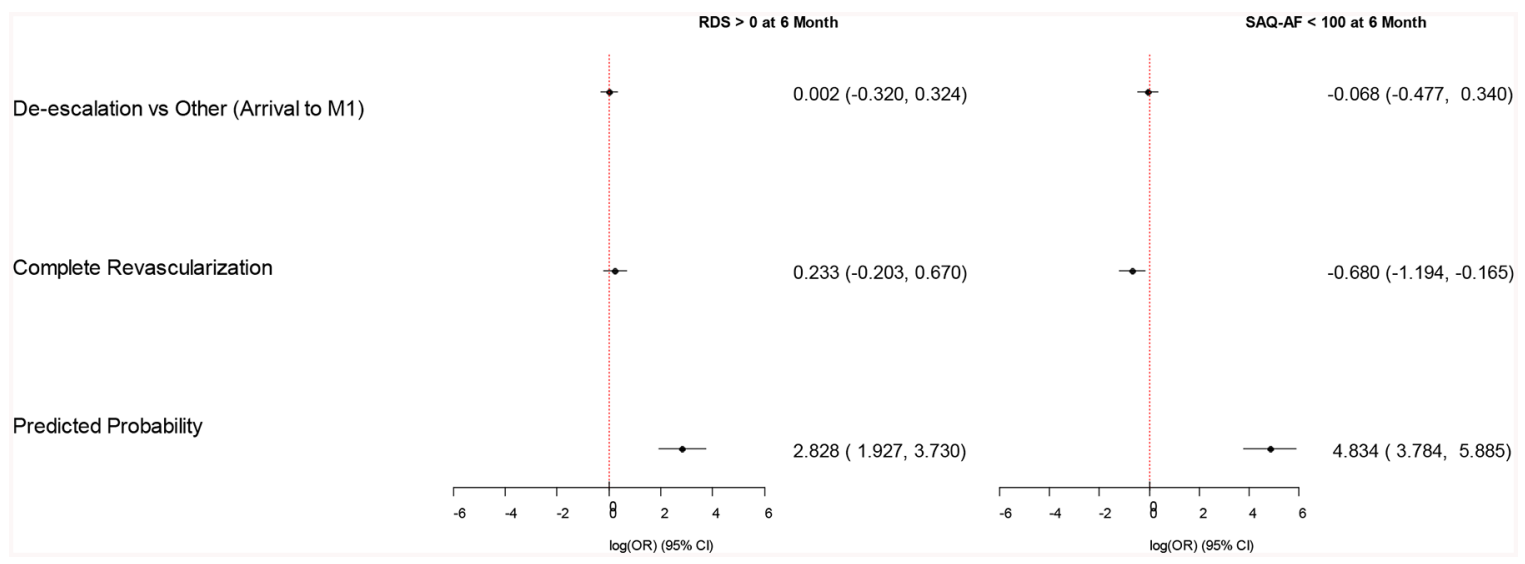

Figure 3. Independent associations of between AAM de-escalation and angina/dyspnea at 6 months after successful CTO PCI.

Adjusted for the pre-procedural risk of predicted residual angina and completeness of revascularization. 
Table 1.

Demographic and clinical characteristics of patients whose AAMs were de-escalated vs not.

\begin{tabular}{|c|c|c|c|c|}
\hline & All Patients n=696 & AAM De-escalation $n=276$ & No AAM De-escalation $n=420$ & P-value \\
\hline Age (y) & $65.6 \pm 10.0$ & $65.6 \pm 10.1$ & $65.6 \pm 9.9$ & 0.974 \\
\hline Male sex & $79.9 \%$ & $79.3 \%$ & $80.2 \%$ & 0.774 \\
\hline White race & $90.4 \%$ & $92.0 \%$ & $89.3 \%$ & 0.230 \\
\hline Current smoker & $12.5 \%$ & $12.0 \%$ & $12.8 \%$ & 0.777 \\
\hline Prior myocardial infarction & $47.1 \%$ & $45.3 \%$ & $48.3 \%$ & 0.431 \\
\hline Prior PCI & $64.2 \%$ & $65.2 \%$ & $63.5 \%$ & 0.641 \\
\hline Prior CABG & $36.8 \%$ & $38.4 \%$ & $35.7 \%$ & 0.471 \\
\hline PAD & $17.1 \%$ & $14.5 \%$ & $18.8 \%$ & 0.139 \\
\hline Prior CVA & $7.6 \%$ & $7.6 \%$ & $7.6 \%$ & 0.995 \\
\hline Heart failure & $21.0 \%$ & $18.1 \%$ & $22.9 \%$ & 0.133 \\
\hline Hypertension & $87.3 \%$ & $88.3 \%$ & $86.6 \%$ & 0.499 \\
\hline Diabetes & $38.2 \%$ & $38.0 \%$ & $38.3 \%$ & 0.938 \\
\hline eGFR & $77.2 \pm 25.0$ & $78.1 \pm 25.4$ & $76.6 \pm 24.7$ & 0.456 \\
\hline Chronic lung disease & $14.8 \%$ & $14.1 \%$ & $15.2 \%$ & 0.687 \\
\hline Predicted risk of residual angina $(\%)$ & $0.2 \pm 0.2$ & $0.3 \pm 0.2$ & $0.2 \pm 0.2$ & 0.002 \\
\hline Physiologic complete revascularization & $85.0 \%$ & $86.5 \%$ & $84.0 \%$ & 0.370 \\
\hline Left ventricular ejection fraction & $51.6 \pm 13.3$ & $52.3 \pm 13.0$ & $51.1 \pm 13.5$ & 0.347 \\
\hline Post-PCI MI & $2.0 \%$ & $2.2 \%$ & $1.9 \%$ & 0.804 \\
\hline CTO lesion length & $60.9 \pm 28.4$ & $58.5 \pm 27.7$ & $62.5 \pm 28.7$ & 0.072 \\
\hline Stent thrombosis & $0 \%$ & $0 \%$ & $0 \%$ & NA \\
\hline \multicolumn{5}{|c|}{ AAM and Health Status Data } \\
\hline \multicolumn{5}{|l|}{ AAM on admission } \\
\hline Any AAM & $100.0 \%$ & $100.0 \%$ & $100.0 \%$ & NA \\
\hline Number of AAM & $1.8 \pm 0.8$ & $2.0 \pm 0.8$ & $1.6 \pm 0.7$ & $<0.001$ \\
\hline Beta-blocker & $92.4 \%$ & $90.9 \%$ & $93.3 \%$ & 0.244 \\
\hline Calcium-channel blocker & $25.9 \%$ & $28.3 \%$ & $24.3 \%$ & 0.241 \\
\hline Long-acting nitrate & $44.4 \%$ & $59.4 \%$ & $34.5 \%$ & $<0.001$ \\
\hline Ranolazine & $15.2 \%$ & $25.0 \%$ & $8.8 \%$ & $<0.001$ \\
\hline \multicolumn{5}{|l|}{ AAM at 1 month } \\
\hline Any AAM & $92.4 \%$ & $80.8 \%$ & $100.0 \%$ & $<0.001$ \\
\hline Number of AAM & $1.6 \pm 0.7$ & $1.5 \pm 0.7$ & $1.6 \pm 0.7$ & 0.003 \\
\hline Beta-blocker & $88.3 \%$ & $79.0 \%$ & $94.3 \%$ & $<0.001$ \\
\hline Calcium-channel blocker & $22.6 \%$ & $18.8 \%$ & $25.0 \%$ & 0.057 \\
\hline Long-acting nitrate & $32.1 \%$ & $25.1 \%$ & $36.7 \%$ & 0.001 \\
\hline Ranolazine & $1.3 \%$ & $1.1 \%$ & $1.4 \%$ & 0.716 \\
\hline \multicolumn{5}{|l|}{ Health status at baseline } \\
\hline SAQ Angina Frequency & $69.0 \pm 27.3$ & $64.9 \pm 29.0$ & $71.7 \pm 25.8$ & 0.001 \\
\hline
\end{tabular}




\begin{tabular}{|l|c|c|c|c|}
\hline & All Patients n=696 & AAM De-escalation n=276 & No AAM De-escalation n=420 & P-value \\
\hline SAQ Physical Limitation & $66.1 \pm 26.0$ & $61.2 \pm 24.3$ & $66.8 \pm 26.2$ & 0.058 \\
\hline SAQ Quality of Life & $48.3 \pm 27.0$ & $44.8 \pm 26.8$ & $50.5 \pm 26.9$ & 0.005 \\
\hline SAQ Summary Score & $60.8 \pm 22.2$ & $58.3 \pm 22.6$ & $62.4 \pm 21.8$ & 0.016 \\
\hline RDS score & $2.0 \pm 1.4$ & $2.1 \pm 1.4$ & $2.0 \pm 1.4$ & 0.532 \\
\hline Health status at 6 months & & & & \\
\hline SAQ Angina Frequency & $92.8 \pm 16.5$ & $91.5 \pm 18.4$ & $93.7 \pm 15.1$ & 0.088 \\
\hline SAQ Physical Limitation & $94.7 \pm 12.8$ & $96.1 \pm 9.2$ & $94.5 \pm 13.2$ & 0.305 \\
\hline SAQ Quality of Life & $80.8 \pm 20.8$ & $79.8 \pm 21.4$ & $81.5 \pm 20.4$ & 0.280 \\
\hline SAQ Summary Score & $88.7 \pm 14.7$ & $87.8 \pm 15.3$ & $89.3 \pm 14.3$ & 0.204 \\
\hline RDS score & $1.0 \pm 1.3$ & $1.1 \pm 1.3$ & $1.0 \pm 1.2$ & 0.242 \\
\hline
\end{tabular}

PCI, percutaneous coronary intervention; CABG, coronary artery bypass graft; NSTEMI, non-ST-elevation myocardial infarction, eGFR, Glomerular filtration rate; MI, myocardial infarction; CTO, chronic total occlusion; CVA, cerebral vascular accident; PAD, peripheral arterial disease; AAM, antianginal medications; SAQ, Seattle Angina Questionnaire; RDS, Rose Dyspnea Scale 
Table 2.

Association of de-escalation in AAM at 1 month after CTO PCI with health status at 6 months

\begin{tabular}{lcc}
\hline & Estimate $(95 \%$ CI) & P-value \\
\hline SAQ Angina Frequency & $-0.86(-3.19$ to 1.46$)$ & 0.466 \\
SAQ Physical Limitation & $1.41(-0.70$ to 3.53$)$ & 0.189 \\
SAQ Quality of Life & $-0.006(-3.03$ to 3.02$)$ & 0.996 \\
SAQ Summary Score & $-0.12(-2.1$ to 1.9$)$ & 0.902 \\
RDS score & $0.003(-0.20$ to 0.20$)$ & 0.999 \\
\hline
\end{tabular}

${ }^{1}$ Adjusted estimate for difference in health status score in patients whose AAMs were de-escalated versus not. Estimates were adjusted for the patient's predicted risk of residual angina after $\mathrm{PCI}$ and complete revascularization. 